\title{
An Analysis of Gastroentereopancreatic Neuroendocrine Tumours at a Tertiary Care Hospital in Southern India
}

\author{
Vinay $\mathbf{K}^{1}$, Balaji Jayasankar ${ }^{2 *}$ and Sonali V Huilgol ${ }^{3}$ \\ ${ }^{1}$ Consultant Surgeon, Columbia Asia Hospital, Mysore, India \\ ${ }^{2}$ Registrar, General and Colorectal Surgery, Maidstone and Tunbridge Wells NHS, \\ United Kingdom \\ ${ }^{3}$ Consultant Surgeon, Mysore Medical College and Research Institute, Mysore, India \\ *Corresponding Author: Balaji Jayasankar, Registrar, General and Colorectal \\ Surgery, Maidstone and Tunbridge Wells NHS, United Kingdom.
}

Received: March 24, 2021

Published: May 28, 2021

(C) All rights are reserved by Balaji

Jayasankar., et al.

\begin{abstract}
Introduction: There is limited data available on epidemiology and clinicopathological patterns of gastroenteropancreatic neuroendocrine tumours (GEP NET) in India. Due to increased availability of advanced endoscopic and radiological imaging, the diagnosis of benign and incidentally identified lesions has also increased over the past decades. Curative surgery is often not feasible since most patients present with metastases at diagnosis. We sought to study the demographics and the behaviour of the tumour.

Methods: The present study is an ambispective study of 25 patients diagnosed to have GEP NETs, treated at over a period of 5 years. Our study focussed on the distribution, epidemiology, treatment and outcomes.

Results: The mean age of presentation was 51 years with a female preponderance of $52 \%$. Most common site of origin was pancreas (56\%) followed by duodenum (32\%). Abdominal pain (68\%) was the most common clinical presentation followed by vomiting (8\%). 17 patients underwent surgery or endoscopic resection. 8 patients underwent medical management. All 25 patients survived and discharged from hospital after respective treatment. There were no deaths recorded in these patients during the hospital stay, however on follow up 5 patients reported mortality in a span of 18 months.

Conclusion: The majority of GEP NET will benefit with a timely surgical intervention along with a sustained medical management. A more robust epidemiological study will help us to understand the burden of this disease better amongst our population.

Keywords: Gastroenteropancreatic Neuroendocrine Tumours (GEP NET); Duodenum; Abdominal Pain
\end{abstract}

\section{Introduction}

Neuroendocrine tumours are a diverse group of tumors that derive from epithelial cells with neuroendocrinal differentiation [1]. The estimated prevalence of neuroendocrine tumors is 1 to 2 cases per 100,000 people, of which Gastrointestinal (GI) tract is the most common site [2]. There is limited data available on epidemiology and clinicopathological patterns of GEP NETs in India. Although Gastroenteropancreatic neuroendocrine tumours (GEP NETs) are generally more indolent than carcinomas, the majority are malignant, showing aggressive tumour behaviour and presenting with metastases at diagnosis. This study was conducted to assess the age distribution, various clinicopathological patterns and management of GEP NETs at a tertiary referral centre in India.
Most neuroendocrine tumours are mainly sporadic, but association with the multiple endocrine neoplasia type 1 syndrome and clustering within families is known [3]. The 5-year survival is mainly associated with stage: $93 \%$ in local disease, $74 \%$ in regional disease and $19 \%$ in metastatic disease with median survival of 33 months $[3,4]$.

Due to increased availability of advanced endoscopic and radiological imaging, the diagnosis of benign and incidentally identified lesions has also increased over the past decades. In terms of symptoms and outcome, clinical behavior of GEP NETs varies strikingly. Overall 5-year survival for GEP NETs varies from 30\% for those that are non-functioning and clinically silent tumors, to $97 \%$ for benign insulinomas [1-4]. 
The diagnosis of GEP NETs is multimodal, based on clinical symptoms, hormone levels, radiological and nuclear imaging, and histological confirmation. Most of them have metastatic disease at diagnosis, with regional or distant metastases observed in $50 \%$ of patients [4].

The primary treatment goal for patients with GEP NETs should be curative surgery. Surgery to remove the primary malignancy and /or local lymph nodes (if affected) is currently the only possible cure and represents traditional first-line therapy. The minimum practical requirements include resectable, well differentiated liver disease with $<5 \%$ mortality, and absence of right heart insufficiency, extra-abdominal metastases and diffuse peritoneal carcinomatosis. However, curative surgery is often not feasible since most patients present with metastases at diagnosis. It is commonly accepted that resection of at least $90 \%$ of the tumor is required to achieve symptom control [5-7].

Approximately $60 \%$ of patients will experience symptom recurrence after surgery and the 5-year survival rate for localized and regional metastases is $35 \%-80 \%[5,7,8]$.

During palliative surgery the primary tumor should also be removed, if possible as debulking may also render medical therapy more effective by decreasing the secretion of bioactive substances. Other modalities of treatment for non-operable cases include somatostatin analogues, chemotherapy and radiotherapy $[5,8,9]$.

\section{Methods}

The present study is an ambispective study of 25 patients diagnosed to have GEP NETs, treated at Kasturba hospital, Manipal over a period of 5 years (June 2010 to June 2015).

\section{Inclusion/exclusion criteria}

All biopsy proven cases of GEP NETs were included, however only biochemical proven/suspected cases were excluded from our study. Study was carried out following approval from institutional ethics committee.

Our study focussed on the distribution and we assessed parameters such as sex distribution, site of tumour origin, clinical presentation, investigations, treatment and outcomes.

\section{Results}

Epidemiologically out of 25 patients, females outnumbered males $(13 ; 52 \%)$ and the mean age of presentation was 51 years.
Most common site of origin was pancreas (14; 56\%) followed by duodenum $(8 ; 32 \%)$ rectum $(2 ; 8 \%)$ and stomach $(1 ; 4 \%)$.

In terms of clinical presentation abdominal pain: 17 (68\%) was the most common followed by vomiting: 2 (8\%), loss of appetite: $2(8 \%)$ and weight loss, diarrhoea, flushing, mass per abdomen: 1 (4\%) each.

For radiological evaluation, all the patients in this study underwent contrast enhanced CT abdomen and pelvis. Endoscopic ultrasound (EUS) was done in 11 patients in addition to CECT.

Out of 25 patients included in the study, 1 patient had estimation of fasting insulin levels in view of recurrent hypoglycemic attacks suggestive of an insulinoma. Fasting insulin levels were elevated $11 \mu \mathrm{U} / \mathrm{mL}$ (normal $<6 \mu \mathrm{U} / \mathrm{mL})$.

Urinary 5-HIAA levels (24 hrs) were estimated in one patient of pancreatic carcinoid in the postoperative follow-up $5 \mathrm{mg} / 24 \mathrm{hrs}$ (normal range: $2-6 \mathrm{mg} / 24 \mathrm{hrs}$ ).

17 patients underwent surgery or endoscopic resection. 8 patients underwent medical management out of which 3 patients received chemotherapy and 5 patients were given palliative symptomatic treatment. The most common surgical procedure performed being whipples pancreaticoduodenectomy (6 patients). Distal pancreatectomy (2 patients), distal pancreatectomy with splenectomy ( 2 patients), enucleation of tumour from pancreas (1 patient), pancreatic head resection (1 patient), distal gastrectomy with gastro-jejunostomy and jejunojejunostomy (1 patient), palliative gastro-jejunostomy and jejunojejunostomy (1 patient), polyp excision (1 patient), snare polypectomy (1 patient) and endoscopic excisional biopsy (1 patient).

Out of 8 patients managed with medical management, 5 patients were given palliative symptomatic treatment and 3 patients were given chemotherapy with doxorubicin and 5 -fluorouracil regimen.

WHO 2010 guidelines classifies neuroendocrine tumours into three grades. All well differentiated neuroendocrine tumours as low grade G1, moderately differentiated tumours as intermediate grade G2 and poorly differentiated tumours as high grade G3. In this study, out of 25 patients - 16 patients belonged to WHO grade 1 (G1), 5 patients belonged to grade 2 (G2) and 4 patients belonged to grade 3 (G3). 
In the present study, all biopsied specimens were tested for panneuroendocrine markers such as chromogranin A, synaptophysin and neuron specific enolase. Out of which, 10 tumours were positive for chromogranin A, 15 tumours were positive for synaptophysin and none of them were positive for neuron specific enolase.

Outcome of patients included in this study was assessed on basis of discharge from hospital after complete recovery. All 25 patients survived and discharged from hospital after respective treatment. There were no deaths recorded in these patients during the hospital stay.

All patients were asked to come for follow-up after 1 month and periodically thereafter. Out of 25 patients, only 20 patients have come for follow up. out of these, 6 patients underwent follow up Gastroscopy and were found to be free of disease. 2 patients underwent colonoscopies which were normal. 12 patients who complained of vague abdominal symptoms underwent ultrasonography which were grossly normal, and patients were treated symptomatically. Others were reassured and kept on further regular follow up.

\section{Follow up}

Out of 25 patients in the present study, 5 patients lost to follow up. out of these 5 patients, 3 patients had undergone symptomatic palliative treatment, 1 patient had undergone palliative gastrojejunostomy and jejunojejunostomy (considered to be inoperable duodenal carcinoid) and 1 patient had undergone distal gastrectomy, gastrojejunostomy and jejunojejunostomy. These patients were called up by phone as part of follow-up. All five patients were reported to have died following a course of treatment elsewhere in an average time of 1.5 years following the diagnosis.

\section{Discussion}

Amarapurkar., et al. (2007) and Xuelong Jiao., et al. (2014) reported there was male preponderance (ratio of 2.5:1) with mean age of $53.01+/-15.13$ years and was male preponderance (ratio of 4:3) with mean age of 56 years respectively. Bruna Estrozi., et al. (2011), there was female preponderance (ratio of 4:3) with mean age of 63.4 years [10-12].

In Amarapurkar., et al. 2007 study, most common presenting symptom was abdominal pain (30 patients, $40.5 \%$ ), followed by miscellaneous (17 patients, 30\%), vomiting (6 patients, $8.1 \%$ ), diarrhea (6 patients, $8.1 \%$ ), loss of appetite (6 patients, $8.1 \%$ ), jaundice ( 4 patients, $5.4 \%$ ), fever ( 4 patients, 5.4\%), GI bleed ( 4 patients, $5.4 \%$ ), carcinoid syndrome (3 patients, $4.1 \%$ ) and loss of weight ( 2 patients, $2.7 \%$ ). In Xuelong Jiao., et al. 2014 study, most common presenting symptom was abdominal pain (49 patients, 33.4\%). In Brazilian neuroendocrine tumor society study conducted by Bruna Estrozi., et al. 2011, most common presenting symptom was abdominal pain (190 patients, $24.5 \%$ ) [10-12].

In present study, most common presenting symptom was abdominal pain (17 patients, 68\%), followed by vomiting ( 2 patients, $8 \%$ ), loss of appetite ( 2 patients, $8 \%$ ), Weight loss, diarrhoea, flushing and mass per abdomen: 1 (4\%) each.

\section{Site of origin}

In Amarapurkar., et al. 2007 study, the commonest site of origin was found to be stomach 22 (30.2\%), followed by pancreas 17 (23.3\%) and duodenum 14 (18.9\%).

In Xuelong Jiao., et al. 2014 study, the commonest site of origin was found to be Pancreas $63(40.9 \%)$ followed by large intestine 43 (27.9\%), Stomach 34 (22.1\%), Small intestine 7 (4.5\%) Biliary tract 4 (2.6\%), Vater's ampulla $2(1.3 \%)$ and Unknown $1(0.6 \%)$.

In Brazilian neuroendocrine tumor society study conducted by Bruna Estrozi., et al. the commonest site of origin was stomach 190 (24.5\%) followed by small intestine 161 (20.8\%), Rectum 159 (20.5\%) pancreas 126 (16.4\%), colon 43 (5.5\%), Appendix 56 (7.3\%) and Esophagus 38 (5\%).

In the present study, commonest site of origin was pancreas 14 (56\%), followed by Duodenum 8 (32\%), Rectum 2 (8\%) and Stomach 1 (4\%).

In the Indian study conducted by Amarapurkar., et al. 2007, 56 patients were grade 1 (G1),14 patients were grade (G2) and 14 patients were grade 3 (G3) tumours.

In the Japanese study conducted by Xuelong Jiao., et al. 2014, 74 patients were grade 1 (G1), 28 patients were grade 2 (G2),42 patients were grade 3 (G3) tumours.

In the Brazilian neuroendocrine tumor society study conducted by Bruna Estrozi., et al. 2011, 566 were grade 1 (G1), 81 patients were grade 2 (G2) and 126 were grade 3 (G3) tumours. 
In the present study 2015, 16 patients were grade 1 (G1) tumours, 5 patients were grade 2 (G2) and 4 patients were grade 3 (G3) tumours.

\section{Conclusion}

Gastroenteropancreatic neuroendocrine tumours are rare and the presentation is varied, thus requiring multi-tune evaluation at a tertiary care centre. The majority of the cases with early detection will benefit with a surgical intervention. But cases presenting with metastasis on diagnosis have a poorer prognosis and would need to be palliated. A more robust epidemiological study will help us to understand the burden of this disease better amongst our population.

\section{Bibliography}

1. Modlin IM., et al. "Gastroenteropancreatic neuroendocrine tumours". Lancet Oncology 9.1 (2008): 61-72.

2. Soga J and Tazawa K. "Pathologic analysis of carcinoids. Histologic reevaluation of 62 cases". Cancer 28.4 (1971): 990.

3. Starker LF and Carling T. "Molecular genetics of gastroenteropancreatic neuroendocrine tumors". Current Opinion in Oncology 21.1 (2009): 29-33.

4. Yao JC., et al. "One hundred years after "carcinoid": epidemiology of and prognostic factors for neuroendocrine tumours in 35,825 cases in the United States". Journal of Clinical Oncology 26.18 (2008): 3063-3072.

5. Oberg K and Jelic S. "Neuroendocrine gastroenteropancreatic tumors: ESMO clinical recommendations for diagnosis, treatment and follow-up". Annals of Oncology 19.2 (2008): ii104ii105.

6. McEntee GP., et al. "Cytoreductive hepatic surgery for neuroendocrine tumors". Surgery 108.6 (1990): 1091-1096.

7. Moller LN., et al. "Somatostatin receptors". Biochimica et Biophysica Acta 1616.1 (2003): 1-84.

8. Kulke MH. "Neuroendocrine tumors: is there a standard treatment?" Gastrointestinal Cancer Research 2.3 (2008): 152-153.
9. Eriksson B., et al. "Consensus guidelines for the management of patients with digestive neuroendocrine tumors-well differentiated jejunal - ileal tumor/carcinoma". Neuroendocrinology 87.1 (2008): 8-19.

10. Amarapurkar DN., et al. "A retrospective clinico-pathological analysis of neuroendocrine tumors of the gastrointestinal tract”. Tropical Gastroenterology 31.2 (2010): 101-104.

11. Jiao X., et al. "Clinicopathological features and survival analysis of gastroenteropancreatic neuroendocrine neoplasms: a retrospective study in a single center of China". Chinese Journal of Cancer Research = Chung-Kuo Yen Cheng Yen Chiu 27.3 (2015): 258-266.

12. Estrozi B and Bacchi CE. "Neuroendocrine tumors involving the gastroenteropancreatic tract: a clinicopathological evaluation of 773 cases". Clinics (Sao Paulo, Brazil) 66.10 (2011): 1671-1675.

Volume 4 Issue 6 June 2021 (c) All rights are reserved by Balaji Jayasankar., et al. 